\title{
Future Directions of Fibrosis in Medicine
}

\author{
Mohnad Abdalla ${ }^{a} \quad$ Amr Ahmed El-Arabey ${ }^{b} \quad$ Xinyi Jiang ${ }^{a}$ \\ aDepartment of Pharmaceutics, Key Laboratory of Chemical Biology (Ministry of Education), School of \\ Pharmaceutical Sciences, Cheeloo College of Medicine, Shandong University, Jinan, China; \\ ${ }^{b}$ Department of Pharmacology and Toxicology, Faculty of Pharmacy, The University of Al-Azhar, Cairo, Egypt
}

Dear Editor,

Fibrosis is described as the overgrowth, hardness, and/ or scarring of different tissues caused by an excess of extracellular matrix components such as collagen. In this regard, fibrosis is the outcome of chronic inflammatory reactions triggered by a number of stimuli such as infections, allergic reactions, autoimmune reactions, radiation, and tissue damage [1].

Moreover, fibrosis is one of the big global strains on health care. The lack of understanding of the fibrotic process pathogenesis, the considerable variety in their etiology and clinical presentations, the lack of relevant and completely validated biomarkers, and, most critically, the present lack of effective disease-modifying therapeutics. Therefore, research has focused on the identification of a new therapeutic target related to human fibrotic disease and the development of successful antifibrotic treatments. To date, only pirfenidone and nintedanib have been approved in some countries to treat fibrotic diseases. Moreover, nintedanib has been approved for the treatment of fibrosing and systemic sclerosis-associated interstitial lung diseases. Consequently, the drug discovery and development remain limited in this critical area and to 1 organ system only, resulting in slow development [2, $3]$. The methodologies of single-cell genomics have produced novel findings that are unattainable before. This area keeps developing rapidly, and the ability to detect the number of omic readouts is a result of emerging technologies (e.g., genomes, proteomes, and transcriptomes) in living cells. The molecular profile is expanding our knowledge of the mechanism of interaction of these populations in situ. Together with global projects, such as the Human Cell Atlas, the convergence and the consolidation of these multimodal single-cell technologies represent an incredible opportunity to decipher the molecular and the cellular mechanisms of fibrosis at an unparalleled resolution, which may help accelerate a new age of accuracy medication in the treatment of fibrotic illnesses. Given the shared pathways across organs that are uncovered by this work, a new revolutionary drug developed for 1 fibrotic disorder is probably useful for most fibrotic diseases [4]. In spite of the remarkable improvement in our comprehension of the pathogenesis of fibrosis during the last few years, several challenges should be addressed to convert this knowledge into successful antifibrotic therapies. For instance, the slow illness development and the patient heterogeneity can be solved by validated and reliable predictors for courses of fibrotic disease. A large compound count versus few patients to be examined can be solved using adaptive and revolution study design and "realworld" substantiation.

Ex vivo tissue culture systems and predictive animal models that lead to the accurate interpretation of new or unprecedented mechanisms from the bench to the bedside karger@karger.com

www.karger.com/ddi

(C) 2021 S. Karger AG, Base

Karger"
Correspondence to:

Mohnad Abdalla, mohnadabdalla200@gmail.com

Xinyi Jiang, xinyijiang@ sdu.edu.cn 
should be developed. The heterogeneity of patients and the slow development of fibrosis lead to difficulty in choosing selection for clinical trials. Therefore, high-risk patients should be identified before their inclusion in the trials, and validated and reliable predictors for fibrotic disease development are required. Patients with fibrotic diseases can be stratified through the tissue sample analysis depending on a mechanistic basis, and antifibrotic drugs can be personalized accordingly [5]. The research should focus on this field. The end points of the study are currently highly variable, and poor sensitivity is required to predict favorable reactions over a limited period, requiring increased patients to be included in clinical trials. As observed in intestinal and liver fibrosis, the last stage for fibrosis clinical trials proceed to develop and can involve a global approach involving patients, researchers, scientists, business leaders, and regulatory partners. In principle, the noninvasive last stage that aligns with clinically relevant findings is needed. Present-day scientists in the field are motivated by the discovery of cutting-edge imaging modalities, such as fibrosis molecular imaging, collagen-targeted positron emission technology imaging, and robust biomarkers, which allow rapid, whole-organ quantitative noninvasive, and longitudinal drug efficacy readings in clinical antifibrotic studies. In addition, the combination of cutting-edge omic processes, like single-cell genomics of fibrosis, and molecular imaging [6], can dramatically enhance patient diagnostics, stratification, prognosis, and cohort enrichment, thereby improving the design of clinical trials and maximizing the number of studies that can be conducted rapidly and effectively. Innovative methods in the design of trials are being introduced to incorporate adaptive techniques. It involves a novel sort of early-phase clinical study, often known as "bucket" or "basket" trial, in which the medicine is evaluated in several baskets at the same time. Depending on the drug's mode of action and the molecular selection of patients, the term "bucket" trial has been used to refer to various scenarios [7].
Substantially, approval procedures are incorporated with the utilization of "bucket" trials involving patients with multiple forms of fibrosis and real-world substantiation. Fibrosis is known as a highly complex condition with several pathways, which when combined, lead to disease progression. Similar to the big developments made in cancer therapy and the effective treatment of hepatitis and HIV, clinical trials and studies evaluating the combination and the integration of drugs for fibrosis treatment have been increasing. Antifibrotic drug combinations are likely to target many orthogonal pathways, comprising a variety of receptors, signaling, and types of cells that have been shown to act in multiple disease states as key drivers of fibrosis. These multifaceted methods can smoothen the process for the delivery of successful antifibrotic treatments in the future.

\section{Conflict of Interest Statement}

The authors declare no competing interests.

\section{Funding Sources}

This work was supported by Shandong Provincial Key Research and Development Program (Major Scientific and Technological Innovation Project) (2019JZZY021013), Shandong Provincial Key Research and Development Program (2019GSF108080), Funds for Youth Interdisciplinary and Innovation Research Groups of Shandong University (2020QNQT003), and Shandong University postdoctoral fellowship to Mohnad Abdalla.

\section{Author Contributions}

M.A. wrote the manuscript and designed the study, A.A.E. and $\mathrm{X}$.J. contributed to manuscript revision. All authors approved the final version.

\section{References}

1 Wynn TA. Cellular and molecular mechanisms of fibrosis. J Pathol. 2008 Jan;214(2): 199-210.

2 Rogliani P, Calzetta L, Cavalli F, Matera MG, Cazzola M. Pirfenidone, nintedanib and Nacetylcysteine for the treatment of idiopathic pulmonary fibrosis: a systematic review and meta-analysis. Pulm Pharmacol Ther. 2016 Oct;40:95-103.

3 Cao J, Cusanovich DA, Ramani V, Aghamirzaie D, Pliner HA, Hill AJ, et al. Joint profiling of chromatin accessibility and gene expression in thousands of single cells. Science. 2018 Sep 28;361(6409):1380-5.

4 Eng CL, Lawson M, Zhu Q, Dries R, Koulena $\mathrm{N}$, Takei $\mathrm{Y}$, et al. Transcriptome-scale superresolved imaging in tissues by RNA seqFISH. Nature. 2019 Apr;568(7751):235-9.

5 Rieder F, Bettenworth D, Ma C, Parker CE, Williamson LA, Nelson SA, et al. An expert consensus to standardise definitions, diagnosis and treatment targets for anti-fibrotic stricture therapies in Crohn's disease. Aliment Pharmacol Ther. 2018 Aug;48(3):34757.

6 Stoeckius M, Hafemeister C, Stephenson W, Houck-Loomis B, Chattopadhyay PK, Swerdlow $\mathrm{H}$, et al. Simultaneous epitope and transcriptome measurement in single cells. Nat Methods. 2017 Sep;14(9):865-8.

7 Cunanan KM, Iasonos A, Shen R, Begg CB, Gönen M. An efficient basket trial design. Stat Med. 2017 May 10;36(10):1568-79. 